\title{
Anesthesia of a patient with Dubowitz syndrome -A case report-
}

\author{
Min Kee Lee, and Yong Seock Lee \\ Department of Anesthesiology and Pain Medicine, Korea Institute of Radiological \& Medical Sciences, Seoul, Korea
}

Dubowitz syndrome is a rare autosomal recessive disorder that leads to growth retardation (intrauterine, postnatal), mental retardation, a peculiar face, microcephaly, behavioral problems and eczema. The peculiar face of individuals with Dubowitz syndrome includes sparse hair and eyebrows, low-set ears, blepharophimosis, bilateral ptosis, a flat nasal bridge with a broad nasal root and micrognathia. Airway management of such individuals might be difficult due to craniofacial anomalies, such as micrognathia, cleft palate, tooth problems and craniocervical anomalies. In addition, anesthetic management may be complicated by other systemic illnesses. We report the uneventful anesthetic management of a 16-year-old girl with Dubowitz syndrome who underwent a total abdominal hysterectomy after a pelvic examination under general anesthesia. We report this case of Dubowitz syndrome with a review of the relevant literature. (Korean J Anesthesiol 2010; 58: 495-499)

Key Words: Airway management, Congenital anomaly, Dubowitz syndrome.

Dubowitz syndrome is a rare, autosomal recessive, genetic disorder. One hundred and forty one cases have been described since the first report by Victor Dubowitz in 1965 [1]. It is characterized by growth retardation (intrauterine, postnatal), characteristic face, microcephaly, mental retardation, behavioral disorder, eczema, etc [2]. The characteristic facial appearance of Dubowitz syndrome include a triangular shaped face, sparse hair, sloping forehead, low set ear, sparse eyebrows and eyelash, blepharophimosis, small palpebral fissures, bilateral ptosis, epicanthus, broad and flat nasal bridge, micrognathia, and dental malocclusion. Systemically, this syndrome has been reported to be associated with cardiovascular, gastrointestinal, urogenital, endocrinological, immunological, hematological, neurological, or musculoskeletal disorders [2]. Anesthetically related clinical features involve craniofacial and craniocervical abnormalities in airway management, as well as cardiovascular, gastrointestinal, and musculoskeletal disorders in anesthetic management. A literature review of this disorder revealed 141 cases but few reports of anesthetic cases. The authors encountered a general anesthesia of a patient with Dubowitz syndrome. We report this case of Dubowitz syndrome with a review of the relevant literature.

\section{Case Report}

A 16-year-old female patient presented with a 3-month history of intermittent vaginal bleeding for treatment at our

Received: August 26, 2009. Revised: September 9, 2009. Accepted: October 1, 2009.

Corresponding author: Yong Seock Lee, M.D., Department of Anesthesiology and Pain Medicine, Korea Institute of Radiological \& Medical Sciences, 215-4, Gongneung-dong, Nowon-gu, Seoul 139-240, Korea. Tel: 82-2-970-2600, Fax: 82-2-970-2161, E-mail: lysjdjn@yahoo.co.kr (c) This is an open-access article distributed under the terms of the Creative Commons Attribution Non-Commercial License (http:// creativecommons.org/licenses/by-nc/3.0/), which permits unrestricted non-commercial use, distribution, and reproduction in any medium, provided the original work is properly cited. 
gynecologic clinic for outpatients. A pelvic examination and vaginal sonography were attempted at the ambulatory care clinic but failed due to lack of patient cooperation caused by her mental retardation. General anesthesia for those tests was then ordered.

According to her medical history, she was born through a cesarean delivery due to hydroamnios at 39 weeks of gestation, and her birth weight was 3,200 g. One month after birth, congenital disorders were suspected and a systemic examination was carried out. As a result, she was diagnosed with Dubowitz syndrome accompanied with an atrial septal defect. The atrial septal defect closed spontaneously at the age of 3 . She had undergone ptosis surgery under general anesthesia at age 4 , and had a history of undergoing general anesthesia for tooth decay treatments. On the pre-anesthetic visit, she was $150 \mathrm{~cm}$ in height, $49 \mathrm{~kg}$ in weight, and showed mild physical growth retardation as well as severe mental retardation with a 1-year old cognitive function. The patient had the characteristic facial appearance, such as microcephaly, sparse hair, sloping forehead, sparse eyebrows and eyelash, blepharophimosis, low set ears, broad and flat nasal bridge, micrognathia, dental malocclusion (Fig. 1), etc. besides eczema on the body and limbs (Fig. 2), which conforms to the common symptoms of Dubowitz syndrome. On an airway evaluation, the extension of the neck was normal with maximal mouth opening. The modified Mallampati Classification was Class I, but micrognathia and uneven teeth were observed. The pre-operative electrocardiogram revealed a premature atrial beat, and the blood test showed a decreased hemoglobin and hematocrit level of $5.4 \mathrm{~g} / \mathrm{dl}$ and $18.7 \%$, respectively, due to vaginal bleeding. The findings of the chest $\mathrm{x}$-ray and urine test were normal. To correct the low hemoglobin level, 3 units of packed red blood cell were transfused to increase the blood level to $9.5 \mathrm{~g} / \mathrm{dl}$ hemoglobin and $28.4 \%$ hematocrit. General anesthesia using mask ventilation was scheduled considering that the length of the test was short and the purpose of the
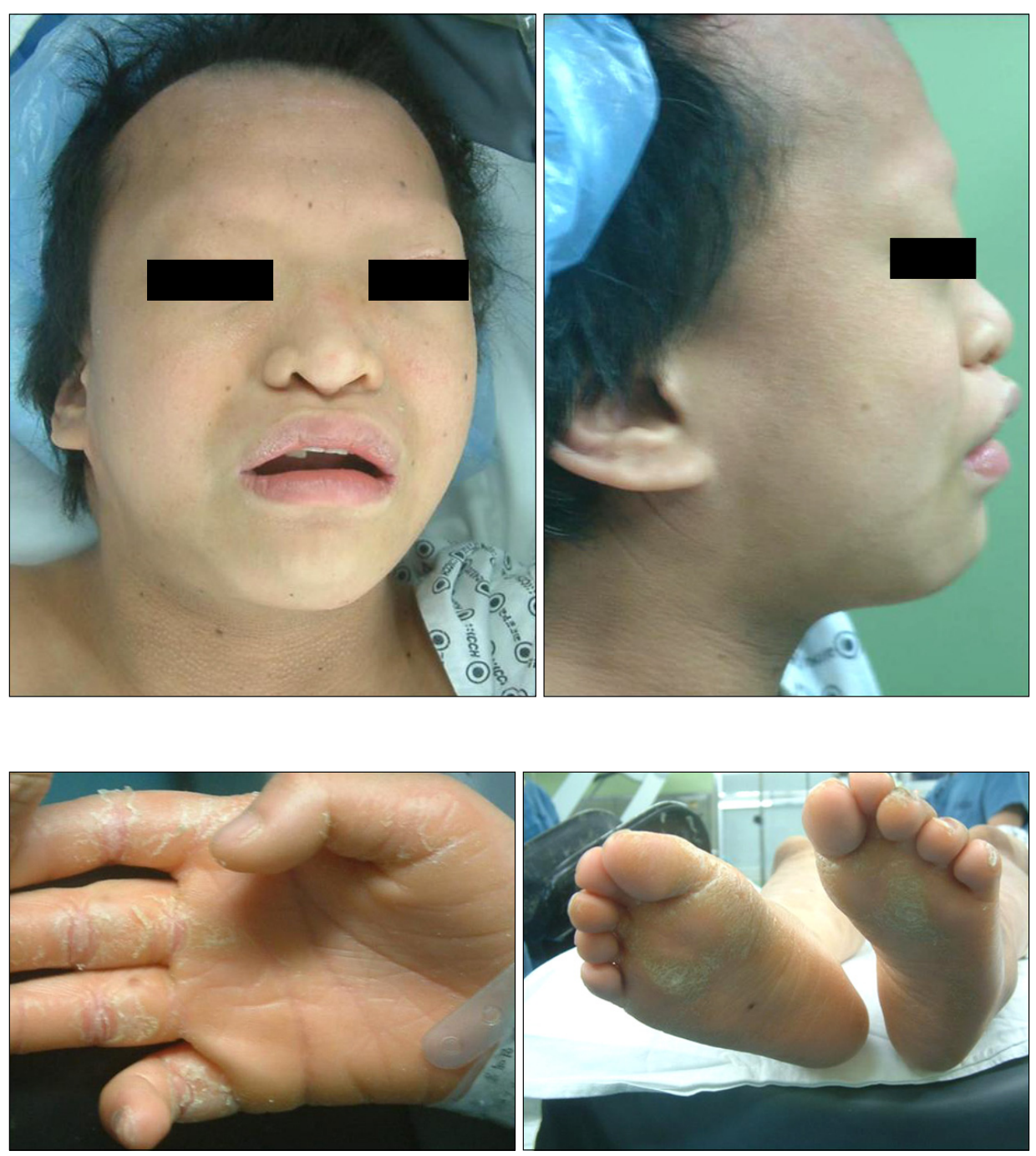

Fig. 1. The picture shows characteristic facial morphology of the patient with Dubowitz syndrome (microcephaly, sparse hair and eyebrows, sloping forehead, low-set ear, flat nasal bridge, micrognathia).

Fig. 2. The picture shows chronic eczema in hands and foots of the patient with Dubowitz syndrome. 
anesthesia was for diagnosis without muscle relaxation.

Without premedication, she was transported to the operating room, where a monitor for the blood pressure, pulse oximetry, electrocariogram was attached to the patient. For the vital signs of pre-anesthesia, she had a heart rate, blood pressure and pulse oxygen saturation of 100 beats/min, 110/65 mmHg and $98 \%$, respectively. The electrocardiogram showed an atrial premature beat at the trigeminy. Mask ventilation with $100 \%$ oxygen was performed after injecting $250 \mathrm{mg}$ of thiopental sodium slowly and a loss of consciousness was confirmed. However, the ventilation did not function well due to an episode of airway obstruction, and an oral airway was inserted. Thereafter, normal ventilation was secured and the anesthesia was maintained with oxygen $2 \mathrm{~L} / \mathrm{min}$, nitrous oxide $2 \mathrm{~L} / \mathrm{min}$, and sevoflurane 2 vol\%. After placing the patient in a lithotomy position, a pelvic examination and transvaginal ultrasound were performed, which indicated cervical myoma and uterine didelphys. A total abdominal hysterectomy was performed to treat the vaginal bleeding. The supply of nitrous oxide was stopped. Ventilation with $100 \%$ oxygen and 5 vol\% sevoflurane was set and $40 \mathrm{mg}$ of rocuronium was administered. After confirming muscle relaxation, endotracheal intubation was performed under a direct laryngoscope. On laryngoscopy, the oral cavity was not narrow, but the larynx was set high to the anterior position, and was classified as grade 2 according to the Cormack-Lehane's classification of direct laryngoscopy grades, in that the anterior portion of the glottis was not visible and only the posterior commissure of the glottis was seen. An endotracheal tube with an internal diameter of $7.0 \mathrm{~mm}$ was intubated at the first attempt. Anesthesia was maintained with oxygen at $2 \mathrm{~L} / \mathrm{min}$, nitrous oxide at $2 \mathrm{~L} / \mathrm{min}$, and sevoflurane $2 \mathrm{vol} \%$, and the ventilation was adjusted with a set of $29-30$ $\mathrm{mmHg}$ of end-tidal carbon dioxide tension. The premature atrial beat disappeared after intubation, and a normal sinus rhythm was observed. The intra-operational vital signs were maintained within the normal limits with a blood pressure, heart rate and pulse oxygen saturation of 100-120/65-80 $\mathrm{mmHg}, 80-100$ beats/min and $99-100 \%$, respectively. As a post-operational procedure, muscle relaxation was reversed with an injection of $15 \mathrm{mg}$ of pyridostigmine and $0.4 \mathrm{mg}$ of glycopyrrolate. The endotracheal tube was extubated after confirming full recovery of spontaneous breathing. The premature atrial beat recurred after extubation but the other vital signs showed normal findings. The total length of time for anesthesia was 2 hours and 10 minutes, and maintenance fluid during operation included $700 \mathrm{ml}$ of Hartmann solution, 300 $\mathrm{ml}$ of a normal saline solution, and 2 units of packed red blood cells. However, the urine output was only $150 \mathrm{ml}$. In the postanesthesia recovery room, $50 \mu \mathrm{g}$ of fentanyl was administered for pain control, while the vital signs were normal except for a premature atrial beat on the electrocardiogram. The patient was monitored for 40 minutes and then transferred to the Ward. On the post-operative day 5 , she was discharged home without complications.

\section{Discussion}

In 1965, Victor Dubowitz first described the congenital abnormalities of a 13-year-old English girl with intrauterine growth retardation, primordially short stature, skin rash, microcephaly, mental retardation, etc [1]. In 1971, Grosse et al. defined this series of anomalies as Dubowitz syndrome [3]. This syndrome is passed down as an autosomal recessive disease. There is no gender predominance. The clinical characteristics include growth retardation (intrauterine or postnatal), mental retardation, behavior disorders (extreme shyness, hyperactivity), high-pitched voice, eczema, etc. whereas the characteristic facial appearances involve microcephaly, triangular shaped face, sparse hair and eyebrows, sloping forehead, small palpebral fissures, bilateral ptosis, epicanthus, broad and flat nasal bridge, submucous cleft palate, micrognathia, and receding chin, etc [2]. Systemically, patients with Dubowitz syndrome are vulnerable to infection, and this syndrome is sometimes accompanied by cardiovascular, gastrointestinal, urogenital, endocrinological, neurological, or musculoskeletal disorders [2]. Dubowitz syndrome is also associated with malignant blood dyscrasia [2]. Despite its low prevalence, this disease has been reported in South and North America, Europe, Asia including the Middle East, etc. Having diverse clinical symptoms, this disorder has been identified as a complicated syndrome with diverse expressions of the genotype. Under the hypothesis of an unstable chromosome, more than two thirds of patients underwent chromosome analysis, but no particularly abnormal findings have been discovered $[2,4]$. Genetically incomplete penetrance or fertilization, and factors other than genes have been suspected $[2,5]$. The karyotype studied until today is normal and the precise pathology of Dubowitz syndrome is unknown. Therefore, there is no specific examination for a diagnosis. Clinical observation of the characteristic appearance, behavior patterns, and accompanied disorders pointing to a diagnosis is the only resort available.

Among the various clinical features of patients with Dubowitz syndrome relevant to anesthesia, the first and foremost to consider is airway management. Dubowitz syndrome may be accompanied by a range of craniofacial such as a cleft palate, micrognathia, receding chin, dental malocclusion, etc. and craniocervical malformations. Swartz et al. reported a case of craniocervical conjugated deformity, in which a Dubowitz patient showed cervical stenosis, subluxation of $\mathrm{C} 1$ due to occipital condyle aplasia, complete dislocation of $\mathrm{C} 1$ due to 
dysplasia of the occipital condyle and cervical vertebra, etc [6]. Takahira et al. observed a case of congenital cervical fusion of C2-C3 [7]. Patients with such deformities might be confronted with danger in airway management including mask ventilation, endotracheal intubation, etc. Endotracheal intubation of patients with an undiagnosed craniocervical malformation can cause serious neurological damage. Therefore, it is essential to make a pre-anesthetic precise evaluation and establish an appropriate anesthetic plan. On the pre-anesthetic visit, our patient showed flexibility on neck movement, which excluded the possibility of craniocervical malformation. She was class 1 according to the modified Malampatti classification, and had micrognathia and an irregular tooth structure. General anesthesia using mask ventilation was scheduled, considering the necessity to prepare for unexpected difficulty in airway management, the short length of the prearranged examination, and the purpose of anesthesia restricted for diagnosis, which did not require muscle relaxation. A laryngeal mask airway and fiberoptic bronchoscope were prepared for immediate use in the case of a failure in airway management. The obstruction of the upper airway upon mask ventilation was assumed to be an obstruction of the oropharynx due to a posterior displacement of the tongue, which was relieved by the oral airway for mask ventilation to resume to normal. After both the pelvic examination and vaginal sonography had been performed under general anesthesia using mask ventilation, the patient was diagnosed with uterine didelphys associated with a cervical myoma. It was decided to treat the vaginal bleeding with a total abdominal hysterectomy. General anesthesia using endotracheal intubation was required for the anesthetic option. Considering that mask ventilation was available, endotracheal intubation was attempted under the guidance of a direct laryngoscope. On laryngoscopy, the oral cavity was not narrow, and was evaluated as grade 2 according to the laryngeal view classification by Cormack-Lehane, where the larynx was set so high to the anterior that the anterior portion of the glottis was not observed and only the posterior commissure of the glottis was visible. Intubation was performed uneventfully at the first attempt.

Another concern to consider with relation to airway management is the muscular hypotonia found in some Dubowitz patients [2]. In addition to the residual anesthetic effect, the symptom may have an effect on post-operative airway management and respiratory function. Our patient did not show any abnormal findings in muscle tone on the pre-anesthetic visit and her respiratory function returned to normal after surgery. For patients with suspected hypotonia, it is essential to conduct a pre-operative test of the muscle tone and evaluate the respiratory function while carefully choosing muscle relaxation and monitoring the muscle tone during surgery.
Gastro-esophageal reflux disease that might be present in some Dubowitz patients [8] should also be taken into consideration for anesthetic management. On general anesthesia for patients with gastro-esophageal reflux disease, it would be appropriate to administer metoclopramide or $\mathrm{H} 2$ receptor blockers pre-anesthetically, to prevent pulmonary aspiration by applying cricoid pressure on mask ventilation, and to refrain from using a laryngeal mask. In the present case, there were no abnormalities indicating gastro-esophageal reflux disease on the pre-anesthetic evaluation, and anesthetic management was carried out without special preparations against its development. Fortunately, the procedure was completed uneventfully without complications. In retrospect, it would be wise and important to anticipate the presence or absence of gastro-esophageal reflux disease, which is not uncommon in Dubowitz syndrome, and to make plans for anesthesia.

Several cardiovascular disorders reported in some Dubowitz patients involve ventricular septal defects, patent ductus arteriosus, atrial septal defect, and aortic stenosis, etc [2]. In addition, abnormalities of tachycardia that defy understanding and right ventricular hypertrophy have been reported [2] Seeburger et al. described a case of a 19-year-old male patient with a myocardial infarction caused by coronary arterial stenosis [9]. In the present case, the electrocardiogram on preanesthetic evaluation revealed a premature atrial beat that did not affect her vital signs before and after surgery. To prepare for an encounter of any cardiovascular disorders not detected on pre-anesthetic evaluation of Dubowitz patients, close history taking on the cardiovascular system and supplementary tests should include electrocardiogram, 24-hour Holter monitoring, echocardiography, stress test, etc.

Some patients of Dubowitz syndrome have been reported to show an abnormal immunological reaction due to a susceptibility to inhaled or dietary allergens that cause eczema, atopic dermatitis, asthma, etc $[4,10,11]$. Our patient had a history of atopic dermatitis and showed an abnormality of severe eczema on the body and limbs, which indicated an abnormal immunological reaction. Nevertheless, she underwent surgery uneventfully without showing a specific allergic reaction to the inter-operatively used anesthetic gas or medication.

Dubowitz patients also vulnerable to infections [2], and malignant blood dyscrasia, such as acute lymphoblastic leukemia [12], non-Hodgkin lymphoma [13], etc., are not rare. In particular, they are susceptible to respiratory infections.

A certain number of musculoskeletal deformity cases have also been observed, including delayed skeletal maturation, craniosynostosis, pectus excavatum, joint hyperextensibility, $5^{\text {th }}$ finger clinodactyly, $2^{\text {nd }}-3^{\text {rd }}$ toe syndactyly, etc [2].

In conclusion, general anesthesia was performed uneventfully in the anesthetic management of a Dubowitz patient, except for 
some difficulty in an early attempt of mask ventilation. This case report suggests that for anesthetic management of patients with Dubowitz syndrome, a thorough pre-anesthetic evaluation, such as indirect laryngoscopy and cervical x-ray, should be performed with an anesthetic plan to secure safe airway management and prepare for possible airway management difficulties due to the craniofacial and craniocervical abnormalities. Furthermore, in order to achieve safe and efficient anesthetic management, it might be necessary to pay close attention to the patient, even in recovery, considering any possible systemic complications occurring during and after surgery.

\section{References}

1. Dubowitz V. Familial low birthweight dwarfism with an unusual facies and a skin eruption. J Med Genet 1965; 42: 12-7.

2. Tsukahara M, Opitz JM. Dubowitz syndrome: review of 141 cases including 36 previously unreported patients. Am J Med Genet 1996; 63: 277-89.

3. Grosse R, Gorlin J, Opitz JM. The Dubowitz syndrome. Z Kinderheilkd 1971; 110: 175-87.

4. Thuret I, Michel G, Philip N, Hairion D, Capodano AM, Perrimond H. Chromosomal instability in two siblings with Dubowitz syndrome. Br J Haematol 1991; 78: 124-5.
5. Ahmad A, Amalfitano A, Chen YT, Kishnani PS, Miller C, Kelley R. Dubowitz syndrome: a defect in the cholesterol biosynthetic pathway? Am J Med Genet 1999; 86: 503-4.

6. Swartz KR, Resnick DK, Iskandar BJ, Wargowski D, Brockmeyer D, Opitz JM. Craniocervical anomalies in Dubowitz syndrome. Three cases and a literature review. Pediatr Neurosurg 2003; 38: 238-43.

7. Takahira S, Kondoh T, Sumi M, Tagawa M, Obatake M, Kinoshita E, et al. Klippel-Feil anomaly in a boy and Dubowitz syndrome with vertebral fusion in his brother: a new variant of Dubowitz syndrome? Am J Med Genet A 2005; 138A: 297-9.

8. Mathieu M, Berquin P, Epelbaum S, Lenaerts C, Piussan C. Dubowitz syndrome. A diagnosis not to be missed. Arch Fr Pediatr 1991; 48: 715-8.

9. Seeburger J, Rastan AJ, Mohr FW. Coronary artery disease: a new manifestation in Dubowitz syndrome. Thorac Cardiovasc Surg 2009; 57: 169-70.

10. Antoniades K, Hatzistilianou M, Pitsavas G, Agouridaki C, Athanassiadou F. Co-existence of Dubowitz and hyper-IgE syndromes: a case report. Eur J Pediatr 1996; 155: 390-2.

11. Hansen KE, Kirkpatrick SJ, Laxova R. Dubowitz syndrome: longterm follow-up of an original patient. Am J Med Genet 1995; 55: 161-4.

12. Gröbe H. Dubowitz syndrome and acute lymphatic leukemia. Monatsschr Kinderheilkd 1983; 131: 467-8.

13. Belohradsky BH, Egger J, Meiswinkel M, Knoop M, Weiss M, Sauer O. Dubowitz syndrome. Ergeb Inn Med Kinderheilkd 1988; 57: 14584. 